\title{
All Male Panels and Other Diversity Considerations for ISPOR
}

\author{
Nancy J. Devlin ${ }^{1,2}$ \\ Published online: 22 July 2019 \\ (c) The Author(s) 2019
}

Diversity and inclusiveness are critically important, and the current gender imbalance at the International Society for Pharmacoeconomics and Outcomes Research (ISPOR) conferences is clearly not acceptable. This is my view-and it is also shared by ISPOR Board of Directors and executive team. ISPOR has a large and very diverse membershipover 20,000 individual and chapter members from around the world - and its activities, including its 'flagship' conferences, should reflect this diversity. The fact they do not means we miss the opportunity to fully benefit from all the health economics and outcomes research (HEOR) expertise available within our membership. As ISPOR incoming President for the 2019-2020 term, I welcome Bouvy and Mujoomdar's [1] note as a further spur to action.

In this short response, I will add to Bouvy and Mujoomdar's analysis, to provide further insight into the issue and the causes of the observed gender imbalance. Actions which are already being taken by ISPOR, and further courses of action being considered, are outlined. Finally, gender imbalance is set in the context of wider diversity issues.

\section{What is Causing the Gender Imbalance at ISPOR Conferences?}

Conference panel sessions (e.g. workshops and issues panels) are initiated by members, who submit abstracts comprising content and proposed panellists.

This comment refers to the article available at https://doi. org/10.1007/s41669-019-0153-0.

This work reflects the views of the author and does not necessarily represent the views of the University of Melbourne or the Office of Health Economics.

Nancy J. Devlin

nancy.devlin@unimelb.edu.au

1 Centre for Health Policy, University of Melbourne, Melbourne, Australia

2 Office of Health Economics, London, UK
Plenary sessions, in contrast, are planned and assembled by conference organising committees, with support from ISPOR staff and leaders. As a result, ISPOR and its leaders have an opportunity to influence the diversity of these plenary panels. Conference programme co-chairs and ISPOR staff have been charged with the goal of ensuring that there are no all-male plenary panels at ISPOR conferences. This will already have been evident in the plenaries at the ISPOR 2019 conference in New Orleans and will be evident again at the ISPOR Europe 2019 conference to take place in Copenhagen in November 2019. Additionally, all possible efforts are being made to ensure that any plenary sessions not involving panels, e.g. key note speakers, also take diversity into account.

This therefore leaves the issue of the panel sessions initiated and submitted by ISPOR membership.

Following the ISPOR Europe 2018 conference, in the light of concerns a number of us had raised, ISPOR undertook an analysis of abstract submissions and acceptances for issue panels - the session type examined by Bouvy and Mujoomdar [1]. This has been updated to include the most recent conference in New Orleans, providing data on the four most recent major (North American or European) conferences, involving the review of 305 submitted sessions and 1170 individual panellists by name (membership data do not include gender). The analysis included both international and European conference issue panels, as the importance of gender diversity applies to all of ISPOR offerings. The percentage of female panellists by submission was examined, since it is a better overall indicator of gender balance than the number of all-male panels per se.

The gender balance in both submitted and accepted abstracts, combined and by conference, is shown in Fig. 1. The results show that the percentage of panellists that are female is nearly identical for submitted versus accepted sessions; this is true whether or not reviewer scores, which are the primary determinant of acceptance, are controlled for. As shown in Fig. 1, the percentage of female panellists was $34.2 \%$ on submitted abstracts and $34.9 \%$ on accepted presentations for the four conferences combined. This allowed 
Fig. 1 Percentage of female panellists in submitted vs accepted issue panel abstracts from ISPOR four most recent international conferences

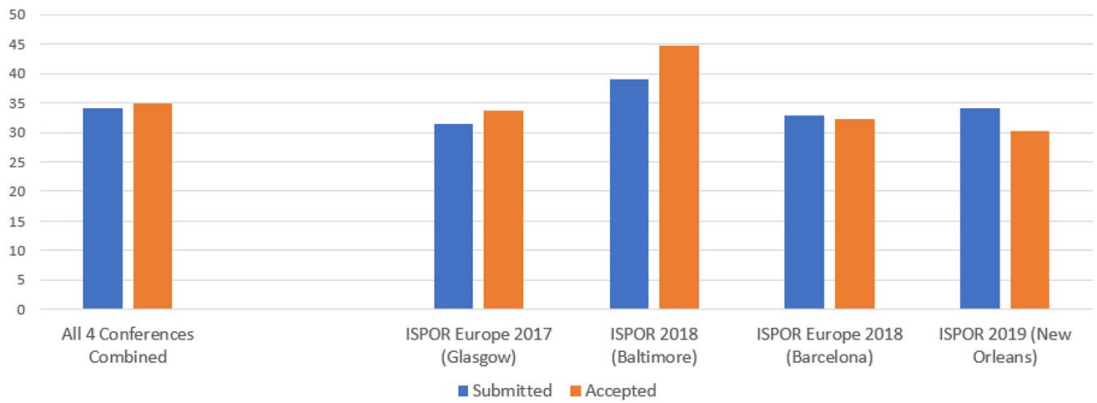

us to reject one possible concern: that the selection process may have been driving or reinforcing gender inequity.

While in one sense this is reassuring, it also means that the gender imbalance which we observe arises from a much more complex set of underlying gender issues. ISPOR is, of course, not alone in this issue: gender imbalance is widely observed in medical and STEM conferences. A recent "Diversity \& Inclusion in Events Report" [2], which analysed more than 60,000 event speakers at events across 23 countries over a 5-year period from 2013 to 2018 , found that only $31 \%$ of speakers were women. A study published on JAMA Network Open, "Trends in the Proportion of Female Speakers at Medical
Conferences in the United States and Canada, 2007 to 2017" [3], examined 701 academic medical conferences and found that only $31.8 \%$ of speakers were female. While we lack accurate statistics, I, like Bouvy and Mujoomdar [1], consider it likely that the HEOR community is approximately gender balanced, and the same is the case with ISPOR membership. Why, then, do we see such marked gender imbalance in the panels submitted from that membership base?

The factors affecting gender imbalance in abstract submission (my thinking about which is inevitably shaped and/ or limited by my own personal experience) include those listed in Box 1.

Box 1: Factors affecting gender imbalance in abstract submissions

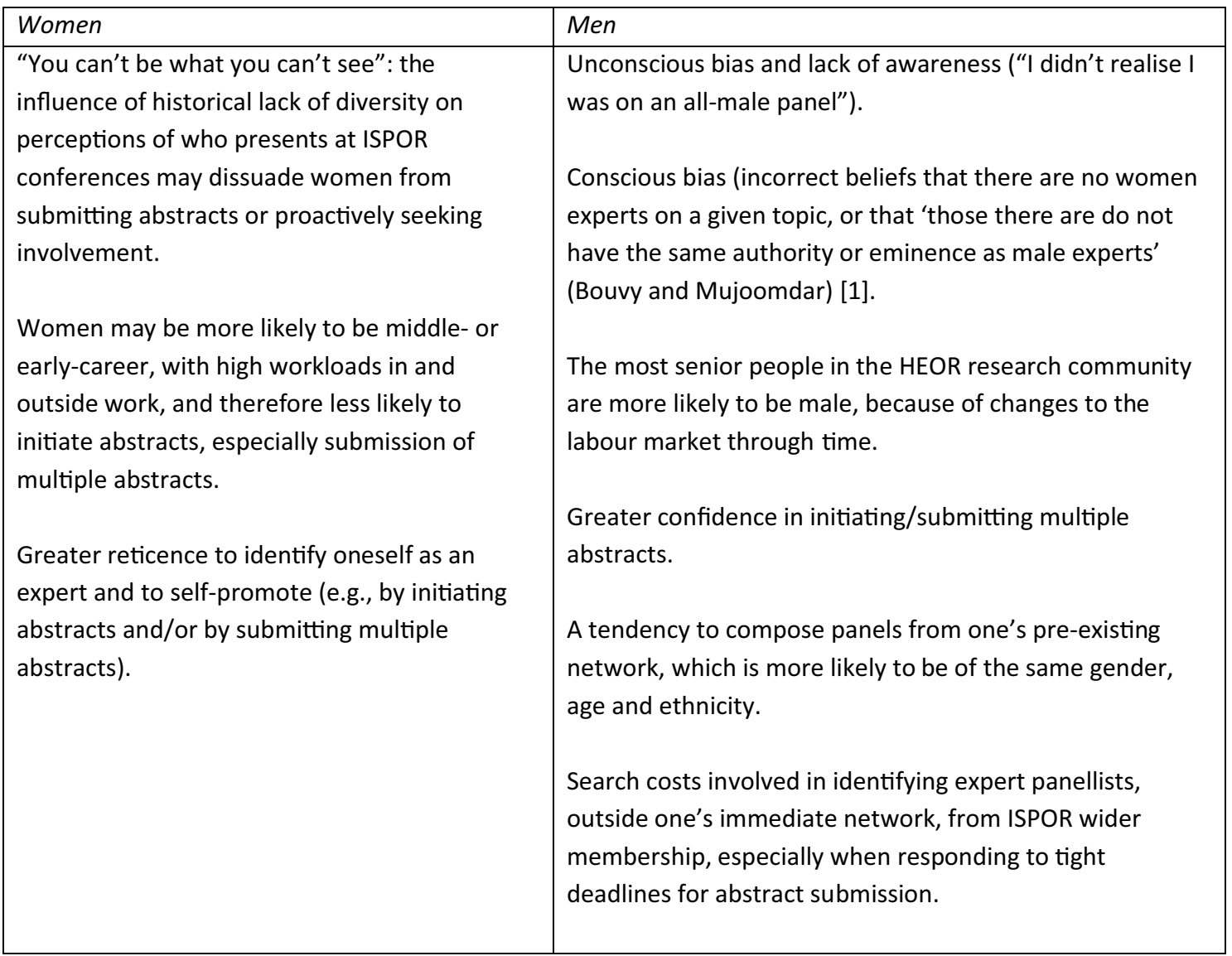


Striving to understand what drives gender imbalance in abstract submissions is important, because it helps ISPOR to identify what combination of strategies is most appropriate to address this.

\section{What is ISPOR Doing to Address the Gender Imbalance in Abstract Submissions?}

There are two principal ways that ISPOR can address this gender imbalance: (1) improve the representation of women in the abstracts submitted, mobilising both men and women to make efforts to this end, and (2) change the selection criteria applied to evaluate abstracts to favour diverse panels.

With respect to (1), actions which ISPOR has already taken include the establishment, in 2017, by Past President Professor Shelby D. Reed, of Women in HEOR [4], which has been active in creating a wide set of networking and mentoring opportunities for women, and is working to educate both men and women about gender issues, including explicit encouragement to women to submit abstracts.

ISPOR online abstract submission form now includes this statement:

ISPOR is strongly committed to diversity. The Society's Strategic Plan and core values embrace excellence through encouragement and acceptance of diverse ideas, cultures, and disciplines. Research submissions are encouraged from all stakeholders and are evaluated based on merit. ISPOR also aims to reflect the diversity of its membership in all endeavours and encourages consideration of diversity in abstract submissions. Diversity dimensions include (but not limited to) gender, career stage, ethnicity, race, education, sexual orientation, region/ geographic location, physical disability, and religion. Additional information can be found at the Society's Diversity Policy.

ISPOR is also currently considering a wide range of further strategies-including ways of raising awareness among men, such as 'nudges' for those who somehow 'missed the memo' and continue to submit all-male panels. We are also considering how to reduce the search costs in identifying content experts among ISPOR large membership, in order to encourage those developing abstracts to "cast the net more widely'.

With respect to (2), the selection criteria are currently under review to identify how best to use these to reinforce the importance of gender balance and diversity more generally. Banning all male panels is an option, but the current approach focuses on raising awareness about why gender balance is important, changing the culture around this, and attempting to bring all members along with us. The selection criteria will be modified to add gender diversity as a factor, but not, for the time being, to create an outright veto for single-sex panels. However, we will monitor the effectiveness of current strategies.

\section{Wider Diversity Issues Affecting HEOR and ISPOR}

Finally, it should be noted that improving the gender balance in ISPOR conferences is one aspect of a wider set of issues relating to diversity. This has been recognised by previous ISPOR presidents and included in strategic planning. Diversity issues featured strongly in the statements I made in my President-Elect speech at ISPOR 2019 in New Orleans (which will be published in the ISPOR Value and Outcomes Spotlight in coming months). Working with CEO Nancy Berg, ISPOR Board of Directors aims to take this agenda forward with greater speed.

Our message is clear: ISPOR is committed to diversity, including (but not restricted to) addressing gender imbalance. It is the right thing to do. I am aware that there are some who think ISPOR is focussing too much on diversity, and not enough on HEOR science, but the reality is that the two are not mutually exclusive. Evidence shows that more diverse organisations have higher performance [5]. ISPOR pursuit of excellence in HEOR will be served by bringing forward and developing HEOR talent from all members, regardless of gender, race, or other characteristics.

To this end, ISPOR Board of Directors has worked with ISPOR executive team to develop a Diversity Policy [6] which was recently approved by the Board. This will be accompanied by a set of actions to achieve those goals. These actions include routine collection and publication of metrics on ISPOR achievement of diversity goals, to which we will be held to account.

ISPOR is an extraordinary and unique organisation both in terms of the breadth of its global membership and in bringing together different stakeholders that include researchers and academics, assessors and regulators, payers and policy makers, the life sciences industry, healthcare providers, and patient engagement organisations. Our aim is to reflect ISPOR diverse membership in its conferences and other activities, and ambitiously, I would like ISPOR to become a beacon of good practice in this respect. I call all ISPOR members to commit to this!

Acknowledgements The author is grateful to Shelby Reed, Betsy Lane and Nancy Berg for helpful comments, and to Richard Willke for preparing the figures. 


\section{Compliance with Ethical Standards}

Funding No funding was received for the work reported in this paper.

Conflict of interest Nancy Devlin is President of ISPOR.

Open Access This article is distributed under the terms of the Creative Commons Attribution-NonCommercial 4.0 International License (http://creativecommons.org/licenses/by-nc/4.0/), which permits any noncommercial use, distribution, and reproduction in any medium, provided you give appropriate credit to the original author(s) and the source, provide a link to the Creative Commons license, and indicate if changes were made.

\section{References}

1. Bouvy JC, Mujoomdar M. All male panels and gender diversity of issue panels and plenary sessions at ISPOR Europe.
Pharmacoecon Open. 2019; https://doi.org/10.1007/s4166 9-019-0153-0.

2. Kumar S (2018) Diversity and inclusion in events report. Bizzabo blog November 2018 https://blog.bizzabo.com/event-gender-diver sity-study (consulted 13th June 2019).

3. Ruzycki S, Fletcher S, Earp M. Trends in the proportion of female speakers at medical conferences in the United States and in Canada, 2007 to 2017. JAMA Netw Open. 2019;2(4):e192103. https ://doi.org/10.1001/jamanetworkopen. https://jamanetwork.com/ journals/jamanetworkopen/fullarticle/2730476.

4. http://www.ispor.org/strategic-initiatives/more/women-in-heor (consulted 3rd July 2019).

5. Hunt V, Layton D, Prince S. Diversity matters. McKinsey. 2015. https://assets.mckinsey.com/ /media/857F440109AA4D13A54D 9C496D86ED58.ashx.

6. ISPOR. Diversity Policy. 2019. https://www.ispor.org/about/oursociety/diversity-policy (consulted 13th June 2019). 